\title{
Marqueurs d'oralité en diachronie : tentative de définition
}

\author{
Pierre VerMander \\ Université Sorbonne Nouvelle Paris 3 \\ pierre.vermander@gmail.com \\ https://orcid.org/0000-0002-2422-432X
}

\section{Resumen}

Este artículo tiene por objeto analizar cómo ha sido tratado el tema de las relaciones entre escritura y oralidad, así como comprender la génesis de los diferentes métodos para su estudio. Este rápido estado de la cuestión permitirá suscitar algunos problemas ligados a varias propiedades de los textos medievales, así como introducir la distancia que queremos adoptar respecto a aquellos métodos de estudio anteriores. Concedemos a la oposición tecnológica una importancia crucial y acudimos al imaginario de los sistemas como herramienta de análisis: ello nos permite llegar a una definición de los marcadores de oralidad como representaciones no equivalentes. Estos marcadores no representan la oralidad porque adopten las propiedades de esta, sino porque la inscriben gráficamente, en virtud de un imaginario de los sistemas de signos.

Palabras clave: oralidad, escritura, oral representado, marcadores de oralidad.

Résumé

Cet article cherche à analyser la façon dont la question des rapports entre écrit et oral a pu être traitée, ainsi qu'à comprendre la genèse des différentes méthodes. Ce rapide état de l'art permettra de soulever quelques problèmes liés à plusieurs propriétés des textes médiévaux ainsi qu'à introduire le léger décalage que nous souhaiterions adopter par rapport à ces approches. En accordant à l'opposition technologique une importance cruciale et à l'aide d'un médiateur (l'imaginaire des systèmes), nous aboutirons à une définition des marqueurs d'oralité comme représentations non ressemblantes : cette catégorie ne représente pas l'oral parce qu'elle lui emprunte ses propriétés mais plutôt en raison à la fois de son inscription graphique et de sa conformité avec un imaginaire des systèmes de signes.

Mots-clés : oral, écrit, oral représenté, marqueurs d'oralité.

\footnotetext{
*Artículo recibido el 15/09/2020, aceptado el 30/03/2021.
} 


\begin{abstract}
This article aims to study orality/writing's distinction as well as trying to understand what lies underneath the way it was dealt with. This summary will allow us to bring up some difficulties relating to some of the medieval text's characteristics and to define the slight shift we would like to carry out. By making the technologic opposition the most crucial property and by instituting a systems' imaginary, we will be able to define orality markers as non-ressembling representations. This category does not represent orality because of it borrowing its features but rather because of its graphicness and alignment with this sign system's imaginary. Keywords: oral, writing, represented orality, orality markers.
\end{abstract}

\title{
1. Introduction
}

Les poncifs ont la vie dure, mais il est un moment où ils méritent d'être abandonnés. Si l'intérêt pour l'oralité ne se dément toujours pas aujourd'hui, il est aussi maintenant difficile de débuter son propos par une remarque liminaire sur l'appétence récente de la linguistique (synchronique ou diachronique) pour l'oral. Nous possédons désormais bien trop d'études sur la parole ou la voix médiévale pour pouvoir se réclamer d'une quelconque originalité.

Il serait d'ailleurs bien plus intéressant de se demander pourquoi une bonne partie des diachroniciens se sont tournés vers l'oral, et si cet intérêt peut être rapporté non pas à une contingence épistémologique mais à l'éclosion d'un désir. Car, du moins pour ces derniers, étudier l'oral tient au départ d'une ambition chimérique : il n'existe - autre poncif - aucune source qui pourrait nous renseigner de façon objective sur la parole médiévale, et le rapport qu'entretient le chercheur avec la voix tient moins de l'enquête que du braconnage. Il s'agit de suivre des traces, signes et pistes laissés par d'autres dans une perspective que l'on ne peut réduire à un rapport de représentation et qu'il nous faut investir afin de les rendre signifiants.

Il est édifiant de constater que cet engouement remonte, à la fois dans le domaine linguistique mais aussi de l'histoire, à peu près au même moment. Dans les années 1970, sous l'influence de Claire Blanche-Benveniste, la langue parlée se voit mettre à l'honneur, et même si Le français parlé : Transcription et édition (Blanche-Benveniste et Jeanjean, 1987) ne traite la question qu'en synchronie, il faut se rappeler que l'ouvrage est préfacé par Jacques Monfrin, alors directeur de l'École nationale des chartes. Ce moment est aussi celui de l'histoire-ethnologie culminant dans Montaillou, village occitan, où l'auteur, dans un avant-propos qui ne saurait aujourd'hui passer au crible de la critique des sources ${ }^{1}$, déclare : "Par chance pour nous, par malchance pour eux,

\footnotetext{
${ }^{1}$ La génération historienne suivante, formée sous l'égide du linguistic turn, reviendra sur les présupposés de l'histoire-ethnologie et diagnostiquera aux textes, a contrario, une extinction de voix. Cf. par exemple Chiffoleau (1990) pour les documents inquisitoires ou Lett (2008) pour les procès de canonisation.
} 
un homme, au XIV ${ }^{\mathrm{e}}$ siècle, a donné la parole aux villageois, et même à tout un village en tant que tel " (Leroy-Ladurie, 1975 : 9). Il faut croiser cet intérêt interdisciplinaire avec le succès de librairie inattendu que fut cet ouvrage pour parvenir à réfuter l'idée d'un simple hasard dans l'arrivée de la voix au sein des études universitaires. Il existe là pour nous une dynamique qu'un savant du premier $\mathrm{XX}^{\mathrm{e}}$ siècle, avant-coureur de Thomas Kuhn, avait nommée un "collectif de pensée »:

Une théorie de la connaissance ne doit pas considérer l'acte cognitif comme une relation binaire entre le sujet et l'objet, entre celui qui connaît et ce qui est à connaître. Parce qu'il est un facteur fondamental de toute nouvelle connaissance, l'état du savoir du moment doit être le troisième terme de cette relation. [...] C'est pourquoi l'acte cognitif n'est en aucun cas le processus individuel d'une "conscience" théorique " existant de toute façon "; il est le résultat d'une activité sociale, puisque l'état des connaissances du moment dépasse les limites imposées à un individu (Fleck, 2008[1935] : 73).

C’est par conséquent dans une communauté mais aussi dans le style de pensée d'une époque qu'il nous faut aborder la question, dans un collectif de pensée, c'est-àdire dans « la communauté de personnes qui échangent des idées ou qui interagissent intellectuellement " étendue au-delà du champ strictement universitaire : "Nous tenons en lui le vecteur du développement historique d'un domaine de pensée, d'un état du savoir déterminé et d'un état de la culture, c'est-à-dire d'un style de pensée particulier» (Fleck, $2008: 74)$.

Pourquoi alors cet intérêt pour l'oral, et pourquoi le trouve-t-on encore davantage dans les travaux des médiévistes ${ }^{2}$ ? Il existe, selon nous, deux types d'explications. La première, positiviste, prendrait en compte le faisceau d'hypothèses permises par la linguistique synchronique : l'ensemble des travaux sur l'oral contemporain ouvrait de nombreux espaces de recherche dès lors que l'on cessait de considérer les textes comme absolument distincts de l'interaction et fonctionnant, à l'inverse, comme des actes communicatifs à part entière ${ }^{3}$; la persistance ou la disparition de certaines catégories grammaticales ou de phénomènes syntaxiques invitait à tracer leur évolution; la nouvelle vision des pratiques d'écriture imposait une relecture des études anciennes quant à la mention de l'événement de parole dans les sources et nécessitait de revoir le rapport entre cet événement et sa traduction graphique ; etc. La seconde hypothèse, fonctionnant de conserve avec la première, tient davantage du négatif : il y a quelque chose de grisant dans le fait de se confronter à l'impossible, mais aussi à ce qui, implacablement,

\footnotetext{
${ }^{2}$ Cf. Anheim (2020 : 18) : "Malgré les travaux d'Arlette Farge ou de Roger Chartier, rien de comparable, en histoire moderne, avec la passion des médiévistes pour la voix et ses métaphores ».

${ }^{3}$ Sur ce point, $c f$. Jacobs et Jucker (1995).
} 
nous indique l'achèvement du passé. C'est d'ailleurs une des leçons d'un congrès récent sur « La voix au Moyen Âge » :

Cette absence est plus cruelle encore du fait que, par nos efforts collectifs, nous pouvons comprendre, à distance, que ce que nous avons perdu était, souvent, ce qui comptait le plus, ces voix emplies de la force de la Création qui, au Moyen Âge, dialoguaient avec les morts, les saints et les oiseaux (Anheim, 2020 : 19).

Il nous faut par conséquent nous interroger sur ce que signifient ces termes (" oral ", " oral représenté ") pour notre période et tenter de les confronter à nos hypothèses. Ce bref état de l'art, on le verra, entraînera la discussion de certains de ses présupposés et nous engagera à définir linguistiquement ce que l'on nomme les «marqueurs d'oralité ", ainsi qu'à préciser, plus globalement, le choix d'une certaine posture vis-à-vis des textes médiévaux mais aussi du Moyen Âge lui-même.

\section{2. État de l'art et discussion}

Les études sur l'oralité médiévale nous paraissent pouvoir être subsumées sous trois modèles théoriques majeurs : celui de l'oral représenté, celui des marqueurs discursifs et celui de la distinction posée par Koch et Oesterreicher (2001).

En fondant le concept d'oral représenté, Marchello-Nizia allait donner naissance à nombre d'études consacrées à la question. La catégorie délimitait un lieu dans le texte où l'on pouvait apercevoir la parole d'un personnage et une langue autre se faire jour :

[Il s'agit de] l'expression qui met l'accent sur la volonté auctoriale de simuler, de mettre en scène du parlé, distingué du narré par des balises spécifiques, et sur le fait que cela se passe à l'écrit. [...] Cette dernière ressource [le discours direct inséré dans la fiction], dans ses spécificités, offre des possibilités non négligeables, en particulier parce que c'est le seul lieu où un contraste entre "oral représenté " et récits écrits de la même main s'offre aussi clairement (Marchello-Nizia, 2014 : 166).

Plutôt que de discuter cette notion ayant le mérite de la clarté et d'une distinction relativement facile, il nous semble plus intéressant d'essayer de comprendre sa genèse. Car il semble que cet oral représenté ne puisse véritablement être dissocié de sa contrepartie : l'apparition de grands corpus de données. La constitution de telles ressources, dont la principale demeure la Base de Français Médiéval (BFM) et à laquelle contribuent nombre de chercheurs ayant repris le concept, permet en effet une révolution dans l'appréhension de la langue médiévale à l'aide de l'encodage des textes en XML-TEI. La possibilité de baliser l'ensemble des passages au discours direct facilite en effet l'extraction de ces ensembles afin de les étudier de façon contrastive avec le reste 
du texte ; en d'autres termes, l'encodage du discours direct constitue la condition de possibilité d'une grammaire de l'oral représenté fondée sur des données empiriques et outillées.

La seconde approche se fonde sur un socle pragmatique, quand la première possédait des fondations syntaxiques. C'est, là encore, l'intérêt de la linguistique pour les "petits mots " du discours (répondant à de nombreuses appellations et que l'on résumera ici sous le terme de marqueurs discursifs) qui semble avoir donné naissance aux études diachroniques. Par analogie avec les analyses contemporaines, et parce que l'on retrouve ces marqueurs discursifs dans les interactions orales, alors leurs équivalents diachroniques se voyaient propulsés au rang de marqueurs d'oralité. Il est cependant à noter que Schourup (1999 : 234), dans une mise au point qu'il jugeait nécessaire, refusait de faire des marqueurs discursifs des formes uniquement orales : "Most forms claimed to be Discourse Markers occur primarily in speech [...] but no principled ground exist on which to deny Discourse Markers status to similar items that are largely found in written discourse ".

Cette « découverte " d'une grammaire de l'oral usant amplement de cette catégorie devait par conséquent faire des émules du moment où l'on considérait, dans la lignée de l'oral représenté, les formes du discours direct comme équivalentes à des « épisodes d'oral représenté ». D'où les nombreuses études sur tel ou tel marqueur discursif : Rodríguez-Somolinos (1995) pour certes et voire, Opperman-Marsaux (2011) pour diva, Guillot (2009) pour ore ou encore Vincensini (2006) pour différentes interjections.

Enfin, la majorité des articles sur la question ne pouvaient éviter ce qui semblait un passage obligé : la mention de l'article publié en 2001 par Koch et Oesterreicher où s'établit une distinction fondamentale entre code et langage, c'est-à-dire entre aspect médial et conceptionnel. S’il y est dit que la distinction médiale est discrète alors que les notions conceptionnelles de parlé et d'écrit se répartissent sur un continuum et que les deux romanistes indiquent que tout langage peut se retrouver dans les deux codes, il n'empêche que ce sont les " affinités » qui semblent les intéresser avant tout. Plus une énonciation satisfait les critères de l'immédiateté et plus elle sera susceptible d'être émise sur le code phonique, et vice versa. Cette attitude modifie leur propos, qui passe alors de linguistique à anthropologique, comme ils semblent d'ailleurs en être conscients : " le continuum communicatif [...] se définit, en dernière analyse, par des données anthropologiques qui sont à la base de toute communication humaine " (Koch et Oesterreicher, 2001 : 585). Mais à ce moment, la distinction discrète posée au départ n'est pas envisagée en tant que telle : « en réduisant la question du média [...] à la notion de code, on n'offre pas à penser l'incidence de la substance sur l'énonciation » (Mahrer, 2014 : 149).

Si ces approches ont déjà produit un bon nombre de résultats, il n’en reste pas moins qu'elles véhiculent parfois l'idée d'une oralité qui «irait de soi »: on passe 
rapidement de l'écrit à l'oral représenté sans s'interroger outre mesure sur le fait qu'il s'agit d'occurrences écrites mais aussi sur la réelle typicité orale des occurrences. Traitant des formes comme ça alors dans les textes, censées marquer l'étonnement du locuteur ainsi que représenter l'oral spontané, Lefeuvre et Tanguy (2012 : 185) remarquent justement qu'elles n'apparaissent que très rarement dans les corpus oraux ; de même, Mahrer, en réaction à la définition de Allons comme "ponctuant typique de l'oral » pour Adam (2011: 155), déclare :

Il semble qu' " oral » vaille ici pour un ensemble de pratiques discursives où le morphème serait surreprésenté. On comprend aisément en tous les cas que "allons " n'est pas typique de l'oral au sens où le serait un intonème (Mahrer, $2014: 100$ ).

Il ne convient pas de déduire de tout cela que la linguistique diachronique se fourvoie lorsqu'elle recherche l'oral représenté. Le fantasme de la reconstruction parasite relativement peu les études, qui insistent souvent dès l'entrée sur la représentation et la fiction d'oral, à l'exemple de Marchello-Nizia (2017: 88) ${ }^{4}$ :

On ne s'intéresse pas à l'exactitude du propos rapporté, ou à sa véridicité, à la manière dont le propos est rapporté, ou encore au fait que le propos ait été " réel » ou " fictif ». On s’intéresse centralement au fait que le lecteur et l'auteur lui-même identifient ces fragments d'écrits comme de l'oral reproduit, et en quelque sorte mis en scène avec ses acteurs propres, bref avec ses spécificités distinctes.

C'est dans cette veine que nous souhaiterions nous insérer : avant même que de classer nos occurrences dans la vaste et instable catégorie des marqueurs d'oralité, il conviendrait de se demander ce qui nous amène à les y placer. Il nous semble nécessaire de nous arrêter sur le moment herméneutique de la construction d'une classe étrangère au système de la langue médiévale afin de se prémunir d'un danger : celui d'une interprétation qui imposerait à une catégorie $a d$ hoc construite depuis notre lieu (équivalente à l'ethnologie faite depuis le fauteuil) un ensemble de propriétés assignées a priori. En revenant aux notions fondamentales qui doivent informer notre perception des textes et notre appréhension des marqueurs d'oralité, nous souhaitons simplement faire un léger pas de côté vis-à-vis des études précédentes, déplacement qui nous permettra de redéfinir de façon satisfaisante la catégorie en recourant à un tiers médiant : celui de l'imaginaire des systèmes.

Les textes médiévaux eux-mêmes avaient déjà pu nous mettre en garde contre une approche qui les prendrait au pied de la lettre, grâce à trois propriétés qui, soit dit

\footnotetext{
${ }^{4}$ Mais $c$ f. cependant Kristol (1993: 4), Wlodarczyk (2005: 100), Guillot (2009 : 267), Glikman et Mazziotta (2013 : 82), Rodríguez-Somolinos (2016: 2) ou Parussa (2018 : 10) qui, insensiblement, semblent parfois basculer vers une sorte de reconstructivisme.
} 
en passant, pourraient tout aussi bien appartenir à l'oral ou au médiéval : l'approximation, l'improvisation et la variation. Outre le fait qu'une approche stricte de l'oral représenté comme lieu du texte délimité par des balises spécifiques et figées négligerait des passages au discours indirect ressemblant fortement à des occurrences repérables dans le discours direct mais objectivement indécidables (exemples 1 et 2), il nous semble qu'une telle approche répondrait de façon malaisée à ce qui, pourtant, se retrouve dans nombre de textes de la pratique (exemples 3 et 4) :

(1) a quoy a dit et respondu que par le dampnement de son ame il ne savoit que c'estoit et n'y fut oncques. (Procès de Jacques d'Armagnac, p. 188)

(2) et, aprés qu'ilz les heust veu, il fit pluseurs sermens en renunçant Dieu qu' il tueroit ledit suppliant et sondit filz. (Lettres de rémission du duc de Lorraine René II, 1. 180)

(3) et, en retournant en sa maison, rencontrast Jannette, femme dudit Perrot, en la rue et luy dist telz motz ou semblables : "Paillarde! tu as dit que ma femme estoit ribaude et que le curé l'avoit chevauchee toute droicte " (Lettres de rémission du duc de Lorraine René II, 1. 60)

(4) dist ces parolles : "Si tu ne fiers, je frapperay ", ou semblables en substances. (Lettres de rémission du duc de Lorraine René II, 1. 51)

Cette utilisation de l'outil graphique, qui vient nous rappeler la disjonction fondamentale à ne pas perdre de vue entre l'écrit et l'oral, peut en réalité être rapportée à une raison de nature historique : ce floutage de l'énonciation ne doit évidemment pas être compris comme une maîtrise approximative de la pratique du témoignage pour les médiévaux mais comme ressortissant à un principe de précaution (Lett, $2008:$ 193, après Mornet, 1988 : 122 et Gauvard, 1991 : 349) marquant une prudence juridique vis-à-vis de ce que le scribe décidait d'écrire dans le cas où il y aurait contestation ${ }^{5}$.

Une autre preuve de la différence existant entre les textes et leur actualisation (qui viendrait par conséquent rectifier notre approche globale des textes afin de faire le départ entre nos sources et la performance, et donc l'utilisation de ces dernières dans une optique de reconstruction) tient à une caractéristique que l'on rencontre de façon flagrante dans le théâtre médiéval mais que l'on pourrait probablement étendre à l'ensemble du massif textuel. Au Concile de Trente (1545-1563), le cardinal Gabriele Paleotti milite pour une prohibition du théâtre en raison de la difficulté d'une censure a priori :

Ça ne suffit pas de dire que d'abord on lise ces comédies pour en enlever ce qu'il y a de mauvais : en pratique ça ne marche pas, parce que toujours ils [les comédiens] y ajoutent des mots ou des

\footnotetext{
${ }^{5}$ On trouve aussi ce principe dans la mention de l'âge des témoins, très souvent accompagnée de formules type "ut dixit, ut asseruit " ou d'une précision comme " et plus, et ultra » dans les procès de canonisation, par exemple. Rappelons aussi que si nos exemples appartiennent aux sources de la pratique, l'usage de l'imprécision se retrouve aussi dans certains textes "littéraires " comme les Quinze joies de mariage (éd. Rychner, 1999) voire dans les Manières de langage (éd. Kristol, 1995).
} 
répliques qui ne sont pas écrites; de plus, il ne mettent rien par écrit, si ce n'est le résumé ou l'argument, et ils font tout le reste all'improviso; vouloir après les condamner pour ce qu'ils disent est très difficile (Taviani, 1969 : 39, apud Smith, Parussa et Halévy, 2014 : 69).

S'il faut évaluer ces propos à l'aune de la situation théâtrale en vigueur dans l'Italie du début du XVI ${ }^{\mathrm{e}}$ siècle, il n'en reste pas moins qu'ils témoignent d'une séparation entre nos documents et la représentation non seulement à un niveau absolu (écrit/oral) mais aussi textuel : une bonne partie des textes que l'on conserve ne peuvent équivaloir parfaitement à ce qui était effectivement joué. On en trouve plusieurs exemples dans des didascalies du type "et stultus loquitur etc." (Moralité de l'homme pécheur, apr. v. 1165, édition dans Bénichou-Samson, 2016) mais c'est le procès-verbal de la représentation du Mystère de saint Martin à Seurre en 1496 qui nous en fournit l'attestation la plus convaincante. Au commencement de la pièce, logiquement ouverte par une diablerie, l'acteur jouant le rôle de Satan est victime d'un léger incident :

Puis après commança a parler Luciffer, pendant lequel parlement celuy qui jouoit le parsonnaige de Sathan, ainsi qu'il volut sortir de son secret par dessoubz terre, le feu se prist a son habit autour des fesses, tellement qu'il fut fort bruslé. Mais il fut si soubdaynement secouru, devestu et rabillé que, sans faire semblant de rien, vint jouer son parsonnaige puis se retira en sa maison [...]. Pour le commancement de l'aprés disnee qui fut a une heure, ledict Sathan revint jouer son parsonnage et pour son excuse dist a Luciffer : Malle mort te puisse avorter, / Paillart, filz de putain coquu / Pour a mal faire t'enorter : / Je me suis tout bruslé le cu. / Et puis parfist son parsonnage pour celle clause et les aultres joueurs ensuivant. (Duplat, in La Vigne (1979: 120 [1496]), f. 260r du ms. Paris, BnF fr. 24332)

Ces vers (la barre oblique indique une virgula dans le manuscrit) ne se retrouvent évidemment pas dans le manuscrit mais nous indiquent, cependant, la distance supplémentaire à calculer, parfois, entre l'écrit, l'oral et sa représentation.

Une dernière propriété médiévale, la variation, pourrait enfin nous amener à revoir des positions peut-être un peu trop assurées. Chose amusante, c'est à la fois une des caractéristiques les plus vantées de la période mais aussi une des moins prises en compte dans les études linguistiques. Comme il est futile de le répéter, on sait que les textes médiévaux ne sont pas analysables à l'aune du ne varietur qui informe notre pensée textuelle, forgée par le moment classique. Dans une analyse de la variation des marqueurs d'oralité dans la tradition de Pathelin (Vermander, à paraître), nous nous étions aperçu que cette catégorie variait peut-être encore plus que les autres (70\%), témoignant là encore de ce floutage inhérent au Moyen Âge. Cette variation, cependant, était 
essentiellement adiaphore mais, de ce fait, pouvait nous amener à considérer comme fondamentales non pas la variance possible pour tel ou tel marqueur mais plutôt l'existence d'une matrice de commutation. En d'autres termes, le fait que certains changements puissent être envisagés comme négligeables au niveau fonctionnel donnait par là une importance cruciale à cette insignifiance en plaçant sur le même plan des lexèmes différents. Si l'on décide de faire de cet avertissement une règle, la perspective d'une étude sémasiologique des marqueurs d'oralité (formes $\rightarrow$ fonctions) se trouve, en quelque sorte, minée de l'intérieur ou se doit, du moins, de composer avec ce que l'on a nommé une "pragmaphilologie ». Ainsi, et s'il est certes nécessaire de fournir des études centrées sur un marqueur en particulier, elles pourraient parfois gagner à prendre en compte ses possibles variations ${ }^{6}$ :

(5) Pathelin (Smith, éd.), v. 334-335:

vrayement il feroit plus de voye / qu'il n'a d'icy a Panpelune (B)

il feroit par dieu plus de voie / qu'il n'a d'icy en Panpelune (V) sainct jehan il feroit plus de voye / qu'il n'y a jusque a Pampelune (LR)

(6) Pathelin (Smith, éd.), v. 180 :

Haa, qué maisnagier vous estes (B)

Hee dieu, quel mesnagier vous estez $(\mathrm{V})$

Enhen, quel mesnaiger vous estes (LR)

On trouve aussi cette variance dans le discours de locuteurs qui l'insèrent dans leur énonciation même ; il semble ainsi que, dans l'exemple (7), les trois occurrences soient considérées comme pragmatiquement égales par Jeanne d'Arc:

(7) Interroguee se, depuis qu'elle est en ceste prison, a point regnoyé ou malgree Dieu :

Répond que non; et que aucunes fois, quand elle dit: Bon gré Dieu, ou: Saint Jehan, ou : Nostre Dame, ceulx qui pevent avoir rapporté, ont mal entendu. (Procès de Jeanne d'Arc, Tisset, éd.: 1960 : 150).

En fin de compte, il semble qu'une approche par trop stricte de la catégorie des marqueurs d'oralité soit incompatible avec leur statut dans nos sources ou témoigne d'une analyse fondée sur des critères qui ne correspondent pas forcément à ceux de la période d'étude. Les leçons que l'on peut tirer de ces propriétés, et qui pourraient être confortées par des remarques d'ordre davantage historique comme le phénomène des chaînes d'écriture éloignant d'autant plus l'événement de parole et le texte qui le consigne $^{7}$, nous invitent par conséquent à porter une attention légèrement différente à nos

${ }^{6}$ Sigles des manuscrits : B = Bigot (Paris, BnF fr. 15080); V = La Vallière (Paris, BnF fr. 25467). Sigles des imprimés : $\mathrm{L}=$ Levet (Paris, BnF Rés. Ye 243) ; $\mathrm{R}=$ Le Roy (Paris, BnF Rés. Yf 417). Nous donnons le vers pour l'édition du texte par Smith (2002).

${ }^{7}$ Pour reprendre un texte déjà mentionné (le procès de Jeanne d'Arc), il convient d'avoir à l'esprit que nous ne conservons, en plus de l'instrument latin, la minute française qu'en raison du procès de réhabilitation. Si l'on retrace chronologiquement le déroulé ayant mené à cette rédaction, on rencontre au 
textes et à la distinction entre écrit et oral, distinction qui, par ailleurs, ne possédait pas le tranchant qu'on lui connaît aujourd'hui mais devait être probablement envisagée par les médiévaux comme un entrelacement :

Si l'on admet que le modèle de toute parole y est celle du Christ, on doit reconnaitre une certaine indistinction ou plutôt une liaison organique et indissociable entre les deux éléments, puisque la parole du Christ est pensée comme parole et en même temps transmise par l'écrit (Gerreau-Jalabert, 1997 : 338).

\section{Choses premières}

Il nous paraît nécessaire de revenir aux propriétés fondamentales opposant l'écrit et l'oral afin de pouvoir constituer une catégorie naviguant entre les deux. Comme on l'a dit précédemment, les définitions des marqueurs d'oralité effectuent souvent un saut épistémologique en négligeant d'expliquer ce qui leur permet de classifier tel ou tel lexème comme appartenant à cette classe. Les distinctions effectuées par Koch et Oesterreicher semblaient aller dans cette direction mais s'arrêtaient, on l'a vu, aux niveaux des affinités. Il convient donc de reprendre la description au niveau technologique des deux systèmes de signes que sont l'écrit et l'oral afin de déterminer ce que l'on nommera leur ergonomie : leurs formes, propriétés et contraintes.

Étant donné que l'écrit et l'oral s'opposent de manière discrète sur le plan technologique, c'est-à-dire des systèmes considérés comme des formes (ce qui, on le verra, n'empêche aucunement leur interaction sur le plan sémiotique), leur confrontation dessine plusieurs paires contrastives qui, à leur tour, entraîneront des attentes et des imaginaires distincts. La distinction fondamentale nous semble se loger au niveau des catégories transcendantales de l'expérience : l'oral est fonction du temps quand l'écrit l'est de l'espace. On ne peut, en toute logique, parler que d'une temporalité de la voix, qui n'a pas cours à l'écrit malgré le principe de linéarité souvent mentionné en linguistique mais appliquant à une figure géométrique une dimension qu'elle ne possède pas en propre. Il n'existe par conséquent aucune propriété technique qui impose une consultation linéaire de l'écrit : nous sommes face à un phénomène proprement simultané, qu'il ne faut ni confondre avec celui de la lecture (qui, soit dit en passant, est assez rarement linéaire) ou de l'écriture (qui demanderait un traitement du geste et non du résultat $\left.^{8}\right)$. L'oral, lui, possède au contraire une durée et, par conséquent, une chrono-

moins cinq étapes supplémentaires : 1) la rédaction des articuli de l'interrogatoire; 2) l'événement de parole à proprement parler (l'interrogatoire) ; 3) les notes prises par deux des trois notaires lors de l'interrogatoire (équivalentes à une sorte de plumitif); 4) la minute française, relation de l'interrogatoire rédigée par les notaire chaque soir après la journée d'enquête ; 5) l'intégration de cette minute au procès de condamnation dont l'instrument est rédigé en latin et qui sera lacéré suite au procès de réhabilitation.

${ }^{8}$ Plus proche en cela du concept de textualisation que l'on peut trouver dans Cislaru et Olive (2018). Notons aussi que cette simultanéité de l'écrit a pu être utilisée dans une visée davantage littéraire : que 
logie ; mais, à l'inverse, il n'est pas durable. En d'autres termes, l'opposition entre ces deux systèmes se résume à celle de la trace écrite face au processus oral, c'est-à-dire à un positionnement vis-à-vis de la rémanence : la trace en est le lieu quand le processus s'éteint dans sa propre profération.

Une telle différence possède des enjeux déterminants que l'on résumera sous le terme d'affordance, i.e. de propriétés construites par l'objet ou l'environnement et non par le sujet ${ }^{9}$. Ainsi, la rémanence permet à son tour une organisation de la distance (alors que l'oral est forcément lié à la co-présence), ce qui entraîne des modifications linguistiques entre les deux systèmes au niveau, par exemple, du marquage du tour de parole : il est possible que l'écrit indique davantage le passage du tour alors que l'oral, en raison de paramètres contextuels (prosodie, kinésique, etc.) dont l'écrit est démuni, ne juge pas nécessaire de déployer un tel arsenal. Cette conséquence se manifeste dans notre littéracie par un arsenal typographique figé mais, pour une période où la ponctuation n'a pas encore reçu une telle fonction, pourrait peut-être se retrouver dans la catégorie des marqueurs d'oralité, comme on le verra infra. Il faut aussi remarquer que les conséquences qu'entraînent les différentes affordances ne portent pas seulement qu'au niveau de la langue; sans reprendre le concept, Morsel en indique la force mesurable historiquement en essayant de repenser le statut même du document dans l'analyse historique (ici des chartes) :

Ce recours à l'écriture sans réponse pourrait (ou devrait) être interprété comme l'appropriation d'une source de pouvoir symbolique plutôt que comme le simple détournement d'une technique, "neutre » en soi, au profit de dominants cherchant des moyens de diffuser leurs ordres (Morsel, $2000: 19$ ).

La communication par le biais d'un média «de masse " brise l'échange en supprimant la possibilité même de réponse et instaure de ce fait un déséquilibre social. (id., p. 18).

Les possibilités de planification ou de manipulation ressortissent elles aussi à la rémanence : à la différence d'un oral tenant toujours de la performance (même lorsqu'il a été préparé), l'écrit est lui toujours pré-visible ${ }^{10}$; de même, en tant que processus non

l'on pense à Mobile de Michel Butor ou aux Cent mille milliards de poèmes de Queneau voire, plus globalement, à « La bibliothèque de Babel » de Borges où écriture et géographie deviennent indissociables.

${ }^{9}$ Le concept d'affordance naît sous la plume de Gibson (1977). Comme on s'en doute, la rémanence n'est pas la seule propriété déterminant l'affordance de l'écrit et de l'oral, mais elle semble bien en être la matrice.

${ }^{10}$ On rappellera les propos de Schegloff (1982: 73), qui consonnent ici avec nos remarques précédentes sur l'improvisation : «Even the wholly prespecified talk, which most approximates the enactment of a cognitive object, must be adapted in its delivery to its occasion, and will certainly have been designed with attention to its recipients and the situation of its delivery in the first place - both aspects of interactional sensitivity ". 
durable, l'oral ne peut être corrigé que vers l'avant quand l'écrit, lui, possède la capacité de se reprendre aussi vers l'arrière. Cette propriété avait d'ailleurs déjà été mentionnée par Barthes et son «bredouillement»:

La parole est irréversible, telle est sa fatalité. Ce qui a été dit ne peut se reprendre, sauf à s'augmenter : corriger, c'est, ici, bizarrement, ajouter. En parlant, je ne puis jamais effacer, gommer, annuler ; tout ce que je puis faire, c'est de dire « j'annule, j'efface, je rectifie », bref de parler encore. Cette très singulière annulation par ajout, je l'appellerai bredouillement (Barthes, 1984 : 99).

Ces affordances distinctes vont alors construire des imaginaires des systèmes, responsables pour nous des effets d'oralité, au niveau sémiotique. Il faut bien avoir ici à l'esprit la différence existant entre technologique et sémiotique : à la différence des propriétés techniques, les aspects sémiotiques ne tiennent pas de contraintes absolues ; à l'opposition discrète se substitue plutôt un continuum ainsi que la possibilité de " se jouer » des caractéristiques. Il nous semble alors retrouver ce que Berrendonner (2004) définissait comme les "contraintes d'optimalité » des systèmes induisant la préférence pour le micro-syntaxique à l'oral et, a contrario, du macro-syntaxique à l'écrit, préférence résumée par les deux versants du "même " énoncé : « les Égyptiens leurs dieux c'est les chats » / "les dieux des Égyptiens sont les chats ». À la différence de propriétés technologiques impossibles à trouver dans certains systèmes (impossible de trouver une rémanence de l'oral ${ }^{11}$ ), rien n'interdit de rencontrer des formes "orales " à l'écrit (la mention de l'exemple supra en est la preuve formelle) ou des formes « écrites » à l'oral ; il n'y a pas de fonctionnement sémiotique en vase clos mais la possibilité de jouer des affordances ou des contraintes d'optimalité afin d'introduire dans un système des éléments qui n'y sont pas standards, que cela soit dans le but de construire un « effet d'oralité " ou non. Il est par conséquent impossible de parler d'un écrit représentant l'oralité comme supplément (au sens classique du terme que l'on retrouve en particulier chez Rousseau ${ }^{12}$ ) ; l'écrit ne représente pas l'oral parce qu'il lui emprunte ses propriétés

\footnotetext{
${ }^{11}$ On pourrait nous objecter que l'on peut maintenant conserver la parole au moyen de différents dispositifs. Mais n'appelle-t-on pas toujours ceci des en-registre-ments ? Il y a, au-delà du jeu sur les mots, une véritable question quant au statut de ces données qui demande probablement un réexamen de ce que l'on entend par écriture (l'écriture alphabétique n'en étant qu'une variété). Certaines propriétés de l'oral, et en particulier l'inscription dans une chronologie inéluctable sont dépassées par les dispositifs de conservation qui font alors passer les sources du côté du pôle de l'écrit (on peut « repasser " une bande mais jamais un discours). Il est par contre intéressant de noter que, dans l'évolution des techniques de communication, un floutage de plus en plus important des deux systèmes est en train de se constituer, la preuve la plus flagrante en étant les applications de messagerie entraînant la destruction des informations après un certain laps de temps, mélangeant traces et processus.

${ }^{12}$ On rappellera que, chez Rousseau, la supplémentarité est toujours la forme de la négativité du mal, qu'il s'agisse de l'écriture ou de la masturbation ( $c f$. Derrida, 1967, et en particulier le chapitre «ce
} 
mais parce qu'il se conforme, dans une certaine mesure, à la représentation grammaticale imaginaire que s'en fait le récepteur. On rencontre alors les propos de Mahrer (2014: 146) :

On ne saurait parler de "marque d'oralité " au sens strict de forme linguistique spécifique à l'énonciation orale, puisque de spécificité au plan formel, il n'y en a point. Si une marque linguistique écrite est considérée comme orale, il faut nécessairement y voir le travail d'un imaginaire de l'oral (et de l'écrit) qui conduit à la reconnaître comme tel.

Il est donc principalement question d'imaginaires, ce qui permet de résoudre les paradoxes apparents mentionnés supra par Lefeuvre et Tanguy (ça alors comme forme presque absente des corpus oraux) ou Mahrer (allons comme " oral ") mais aussi ceux de la sur-représentation de marqueurs d'oralité au début du tour de parole à l'écrit ou la sous-représentation graphique de phénomènes envahissants au plan phonique (e.g. les marqueurs de disfluence). Toutefois, là encore, il faut se rappeler que ces interpénétrations ne sont pas impossibles; elles peuvent, en fonction du moment ou du lieu de production être si peu probables qu'on pourrait en venir à les considérer comme nulles mais on ne peut en aucun cas les considérer comme improductibles. La volonté $\mathrm{du}$ « tout transcrire » a pu intégrer dans notre littérature de plus en plus de marques de l'oral que les écrits antérieurs auraient irrémédiablement bannies ; c'est l'exemple de Jucker (2015) étudiant les formes phatiques uh et um dans la fiction contemporaine. Les linguistes diachroniciens sont bien plus démunis mais il ne faut jamais perdre espoir. Nous en voulons pour preuve deux occurrences équivalentes à des quasi-hapax aux niveaux lexical et syntaxique : celui d'une forme très peu attestée dans nos sources (Hem, ayant par la suite donné lieu au hemmage) et d'une structure en escalier typique des interactions orales en raison d'un recours à la micro-syntaxe. Le premier exemple se trouve dans les Propos rustiques de Noël du Fail (éd. Pérouse et Dubuis, 1994) où Mistoudin conte à son frère Brelin le récit d'une embuscade lui ayant fait perdre la face :

dangereux supplément ... »). Cf. les chapitres V et VI de l'Essai sur l'origine des langues (éd. Starobinski, 1990), ainsi que le dernier ("Rapports des langues aux Gouvernemens ») : "Les sociétés ont pris leurs dernieres formes; on n'y change plus rien qu'avec du canon et des écus, et comme on n'a plus rien à dire au peuple sinon, donnez de l'argent, on le dit avec des placards au coin des rües ou des soldats dans les maisons ; il ne faut assembler personne pour cela : au contraire, il faut tenir les sujets épars; c'est la premiére maxime de la politique moderne ». 
(8) Luy dist outre qu'il estoit deliberé s'en venger par un moyen qu'il luy diroit, mais qu'il fust assis et à son aise, et qu'il luy pardonnast s'il estoit Hem : car trop estoit faché de l'offense. (Propos rustiques, p. $128^{13}$ ).

Si l'on rencontre dans les textes du moyen français diverses occurrences des formes Hen, Hem, aucune cependant ne fonctionne de la même façon que celle-ci et il se pourrait bien qu'il faille y voir une attestation du hemmage qu'on ne retrouvera graphié ainsi que bien plus tard dans les documents écrits ${ }^{14}$.

C'est dans le procès de Jeanne d'Arc que se loge la seconde occurrence dont on ne peut avec certitude déterminer l'origine. Alors que l'ensemble du procès est structuré par une alternance de paires adjacentes question-réponse relativement standardisées ( $c f$. exemple 9), un passage semble détonner par rapport au reste :

(9) Interroguee se les compaignons de guerre faisoient point mectre en leurs pennonceaulx Ihesus Maria:

Repond : Par ma foy, je n'en sçay rien. (Procès de Jeanne d'Arc, Tisset, éd.: 1960 : 97).

(10) Dit, quant a la paix, dit, quant au duc de Bourgongne, elle l'a requis, le duc de Bourgongne, par lectres et a ses ambassadeurs, que il y eust paix. Quant aux Angloys, la paix qu'il y fault, c'est qui s'en voysent en leurs pays, en Angleterre. (Procès de Jeanne d'Arc, Tisset, éd.: 1960 : 215).

Le statut de cette réponse diffère à un tel point des autres qu'il nous a semblé préférable de lui appliquer une " mise en grille ", méthode préconisée par la linguistique de l'oral contemporaine et définie ainsi par Blanche-Benveniste et Jeanjean (1987: 165) : "Le mode de lecture consiste à lire chaque ligne l'une après l'autre, quelle que soit leur étendue. Les colonnes verticales qui apparaissent indiquent le remplissage d'un même emplacement syntaxique ". Force est de constater la similitude entre notre occurrence habillée à la mode du GARS et la banalité de ce phénomène dans les interactions orales (exemples 11 et 12) :

\footnotetext{
${ }^{13}$ Nous avons modifié ici l'édition afin de supprimer les interventions et de rétablir le texte tel qu'on le trouve dans l'imprimé de 1549 (Paris, BnF, Arsenal, Rés. 8-BL-30498). Les éditeurs avaient par exemple introduit avant le marqueur des points de suspension et supprimé les deux points suivants.

${ }^{14}$ Ce qui n'empêche par contre pas le hemmage de se retrouver sous d'autres formes (parfois très proches), et en particulier dans l'œuvre de Rabelais, ayant par ailleurs fortement influencé celle de Bonaventure des Périers. Ainsi de la harangue de Maistre Janotus de Bragmardo à Gargantua, lui demandant le retour des cloches de Notre-Dame dans un discours qui semble avoir du mal à se mettre en route : "Ehen, hen, hen! Mna dies, Monsieur, mna dies, et vobis, Messieurs. Ce ne seroyt que bon que nous rendissiez nos cloches, car elles nous font bien besoing. Hen, hen, hasch ! Nous en avions bien aultrefoys refusé de bon argent de ceulx de Londres en Cahors, [...] ». On trouvera, à la fin de la prière, une nouvelle gradation lorsque le magister s'étrangle dans son jargon scolastico-comique : " [...] qui vivit et regnat per omnia secula seculorum, amen. Hen, hasch, ehasch, grehenhasch!" (Rabelais, Gargantua, chap. XIX, Boulenger éd., 1955).
} 
(11) vous savez que on a euh avec le les postes nous avons euh installé fait installer un certain nombre de cabines. (Blanche-Benveniste et Jeanjean, $1987: 151)$.

(12) or de ce point de vue je voudrais tout de même insister sur un point que j'ai été amené à faire valoir dans cette enceinte, que j'ai été amené à faire valoir dans l'enceinte de feu le comité de coordina- ... de heu standardisation. (Blanche-Benveniste et Jeanjean, 1987: 153).

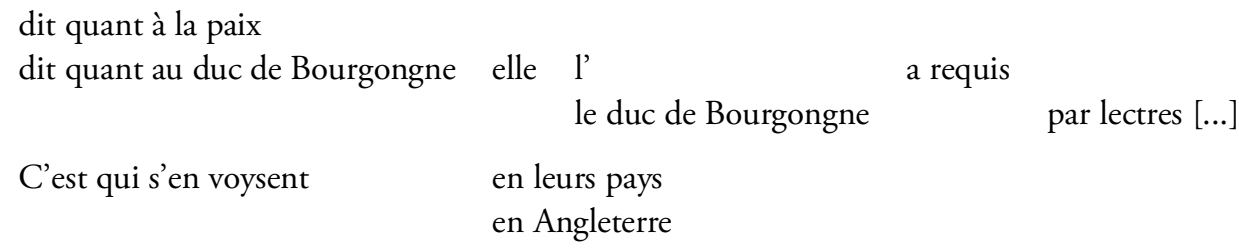

Il est cependant impossible de dire s'il s'agit ici d'une inattention du copiste, d'une prise de note rapide lors de l'interrogatoire qui n'aurait pas été formalisée ou d'une structure que l'on rencontre seulement très rarement. Il semble toutefois à peu près certain que l'on ne saurait analyser cela comme un " effet d'oral » voulu mais plutôt comme une utilisation de l'écrit selon une affordance penchant davantage du côté de l'oral.

\section{Marqueurs d'oralité}

C'est donc pour nous l'interaction sémiotique entre l'écrit et l'oral ainsi que leurs imaginaires (dépendant en partie de l'affordance de ces systèmes) qui, en instituant des conditions de possibilités ou d'impossibilités, de préférences ou de non préférences et des attentes linguistiques différentes, sont à la base de l'effet d'oral nous amenant à placer certaines formes graphiques dans la catégorie des marqueurs d'oralité. Parce que l'écrit, comme on l'a dit, ne représente pas l'oral dans une logique de supplémentarité mais plutôt à l'aide d'une certaine adéquation avec l'imaginaire que s'en fait le récepteur, les marqueurs d'oralité n'ont pas pour propriété fondamentale de se trouver effectivement dans les données naturelles. Pour le dire autrement, ces marqueurs nous semblent ressortir d'une représentation non ressemblante.

C'est, paradoxalement, l'inscription graphique de ces marqueurs qui nous autorise à leur octroyer un statut " oral ": dans notre perspective, les marqueurs d'oralité ne le sont que parce qu'ils sont écrits. Là encore, ceci n'est pas sans empêcher de supposer une parenté avec un oral "réel », à condition de ne pas en faire la clef d'analyse. Il convient forcément de naviguer selon un bon sens résumé, par exemple, dans les propos de Short et al. (1997: 221) : "Speech report must be the report of something ». Le fait qu'un écrit ait besoin d'une réception afin de faire sens exige qu'existe une certaine coïncidence entre les imaginaires des différents protagonistes de l'interaction et la langue elle-même, ce que déclaraient, en termes plus généraux à propos des textes de théâtre, Culpeper et Kytö $(2010: 221)$ : 
All this does not mean that Play-texts in general have nothing to do with naturally occurring conversation. They clearly have enough points of similarity to suggest to the audience that the use of knowledge about conversation in their interpretative procedures is appropriate: a play can hardly proceed without assumptions that the reader/audience knows something about turn-taking, how conversations begin and end, how people greet and part, how speech act are formed to do things, how politeness is signalled, and so on. And playwrights exploit this knowledge.

S'il faut donc refuser une construction des marqueurs d'oralité absolument éloignée de la langue orale, il convient aussi de ne pas perdre de vue la distance entre les deux systèmes ainsi que l'importance jouée par leurs imaginaires.

On voit par là ce qui distingue cette approche des autres modèles discutés précédemment : à la différence d'une logique de l'oral représenté comme lieu du texte autorisant une analyse comparée, notre perspective est davantage représentationnelle. Elle n'a pas pour but d'aboutir à une grammaire comparée de l'oral représenté et de l'écrit mais de comprendre en quoi certains lexèmes ou parties d'un texte peuvent venir stimuler nos représentations imaginaires et aboutir à classer telle ou telle occurrence comme marqueur d'oralité. Il y a donc nécessairement un investissement du chercheur qui s'opposerait, dans la perspective d'une approche grammaticale, à une (prétendue) objectivité du nombre : sans refuser sa pertinence à l'approche contrastive de l'oral représenté, il faut tout de même y reconnaître le désir d'une mise en chiffre des textes où le numérique équivaudrait à l'objectif et, surtout, viendrait compenser la faiblesse inhérente de toute linguistique qui ne peut qu'employer le langage pour parler du langage. Si ce déchiffrement a déjà pu donner bon nombre de résultats, il nous paraît toutefois nécessaire de ne pas en faire l'unique perspective d'analyse. En un sens, nous nous proposons de construire ce que l'on ne peut nommer qu'à l'aide d'une formule paradoxale : un oral médiéval contemporain. Cette pratique d'une intuition contrôlée se retrouve depuis quelques temps dans le champ historique, que cela soit pour la Grèce antique (l'" anachronisme contrôlé " de Loraux, 2005 [1993] ${ }^{15}$ ), la Révolution française (Wahnich, 2009) ou bien la période médiévale, à l'exemple du travail d'Offenstadt (2013 : 25) sur Jean de Gascogne, crieur au XVe siècle :

Nous serons, parfois, amenés à travailler sur des hypothèses fragiles. C'est-à-dire à raisonner à partir de fragments, de quelques mots, de l'absence de documents ou d'absences dans les documents. Mais constituer une hypothèse fragile ne signifie pas raisonner de manière purement spéculative ou dans une forme de vide peuplé par la seule imagination du chercheur. Nous

${ }^{15}$ Cf. par exemple Loraux (2005 : 129) : "Car l'anachronisme s'impose dès lors que, pour un historien de l'Antiquité, le présent est le plus efficace des moteurs de la pulsion de comprendre ». 
entendons que ces propositions s'inscrivent dans les possibles de la période et du milieu social de Jean, qu'elles soient au plus près des possibles attestés par ailleurs.

Un des avantages de cette méthode est qu'en refusant de faire de la représentation de l'oral une finalité des marqueurs d'oralité, elle permet de les appréhender comme des occasions d'étude, à l'aune de leur inscription graphique. En ne forçant pas l'adéquation de l'objet et l'intention de le faire exister, elle rejoint les propos de Schmid et Mambrini-Doudet (2019: 35) et refuse le paralogisme qui voudrait que les marqueurs d'oralité représentent l'oral parce qu'ils appartiennent à la catégorie des marqueurs d'oralité.

Ainsi, en reprenant la question de la présence de marqueurs d'oralité à l'ouverture de parole dans nos textes, il est préférable d'envisager le problème en essayant avant tout de comprendre la raison de ce phénomène avant que d'essayer de le faire équivaloir à un prétendu oral réel. Cette façon de faire nous semble avoir déjà été prônée par Zumthor (1987 : 24), lors de sa relecture des travaux de Jauss :

Je réfere à un certain comportement intellectuel plus qu'à une méthode : accorder à une esthétique de l'effet produit la prééminence sur une esthétique de la production; fonder la démarche critique sur la considération de ce que fut l'" horizon d'attente " du public premier de l'oeuvre; tenir compte d'abord, pour définir celle-ci en tant qu'objet d'art, de la nature et de l'intensité de son effet sur le public ; veiller à toujours poser, dans la mesure du possible, les questions auxquelles l'oeuvre répondait de son temps, avant celles que nous lui posons aujourd'hui.

Nombre de séquences dans nos textes possèdent en effet un taux de présence d'un marqueur d'oralité initial (interjection, adverbe ou coordonnant pragmatique, jurement) anormalement élevé : près de $85 \%$ des tours sont débutés ainsi dans certains passages des Quinze joies de mariage et l'on peut trouver de tels ratios pour d'autres types de textes, comme les Manières de langage. Lorsque l'on compare ces chiffres à des transcriptions de conversations naturelles (qu'il s'agisse d'exemples tirés des analystes de la conversation américains ou de données issues de corpus oraux français comme le CFPP), la sur-représentation littéraire (ou, plus globalement, graphique) se fait nettement sentir. Mais, comme on l'a dit, analyser ces disparités à l'aune des deux systèmes de l'oral et de l'écrit n'aurait pas grand intérêt ; si l'écrit fait ici preuve d'un emploi plus important des marqueurs à cette position, il nous faut tenter de comprendre ce fait comme faisant sens dans son propre système. Cette présence de marqueurs d'oralité contraste d'ailleurs aussi avec les textes postérieurs, où la fréquence des marqueurs recule une fois passés les premiers stades de l'échange. Comme il n'est pas ici question de se pencher de façon approfondie sur cette question, nous préférons insister sur un exemple illustrant à la fois l'emploi de cette catégorie par les médiévaux mais aussi ses 
répercussions éditoriales (c'est-à-dire sur le style de pensée de notre époque). Comme on le sait, les Manières de langage consignent des dialogues entre différents protagonistes utilisés à des fins didactiques pour l'enseignement du français en Angleterre aux XIV et $\mathrm{XV}^{\mathrm{e}}$ siècle. Il s'agit ici d'une interaction entre un vénitien et un français (respectivement $\mathrm{V}$ et $\mathrm{F}$, ces appellations de notre fait ayant pour but de mettre davantage en valeur le propos) :

(13) F - Beau sir, dont venez vous, se vous plest? Vel sic : De quel part venez vous, mon tresdoulx amy, mes q'il ne vous displese?

$\mathrm{V}$ - Vraiment, sir, je vien tout droit de Venys.

F - Hé, mon amy, c'est un ville de Lumbardie!

V - Oil vraiment, beau sir, si est.

F - Par mon serment, mon tresgentil sir, j'en ay grant joy de vous que vous estez si bien travaillé, depuis que vous estes si joefnes, quar je pense bien que vous n'avez unquore .xxx. ans.

$\mathrm{V}-\mathrm{Si}$ ay je vraiment et plus, mais pur ce que je su bien sains et joliet au cuer, la mercy Dieu, l'en me dit que je su plus joefnes que je ne su.

F - Ore sir, est Venys une beale citee ?

$\mathrm{V}$ - Oil dea, et le plus noble porte que est en tout le monde, sicom l'en m'a dit en cele pais qu'ont travaillé

partout.

$\mathrm{V}$ - Et de que pais estes vous, beau sir, mes q'il ne vous displese?

F - Veraiment, sir, je su de France

$\mathrm{V}-$ Et de quele ville, se vous plest ?

F - De Parys, sir. (Manières de langage 1396, éd. Kristol, 1995 : 32) 


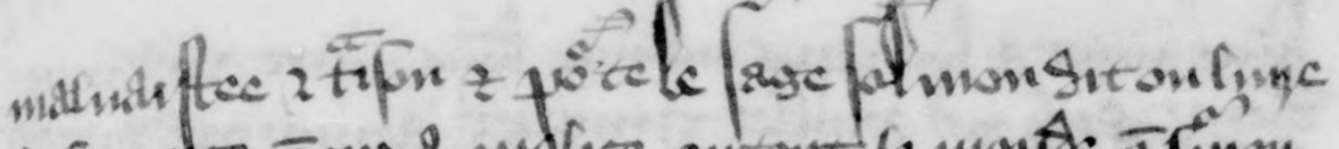

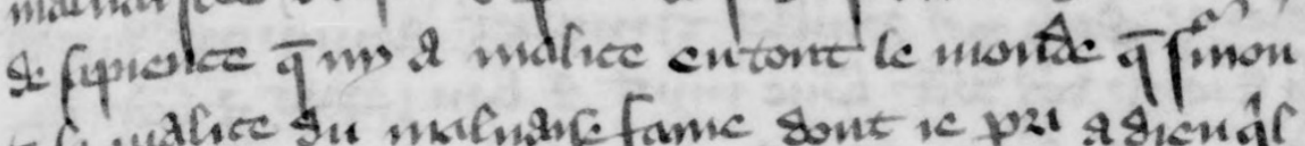

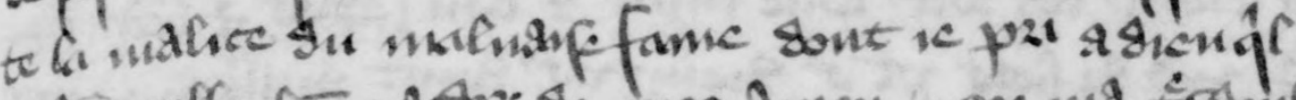

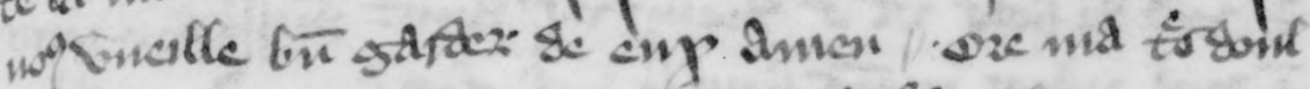

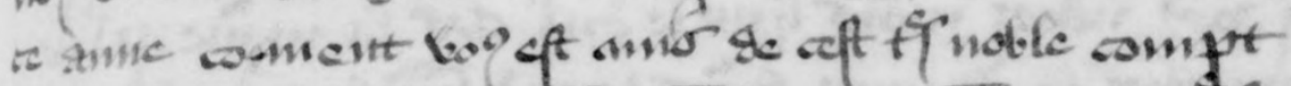

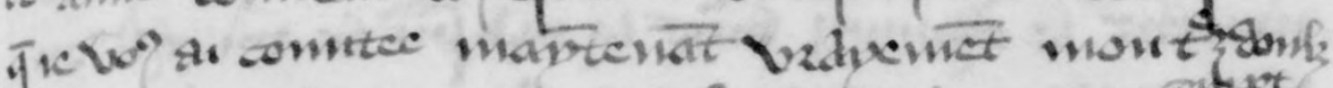

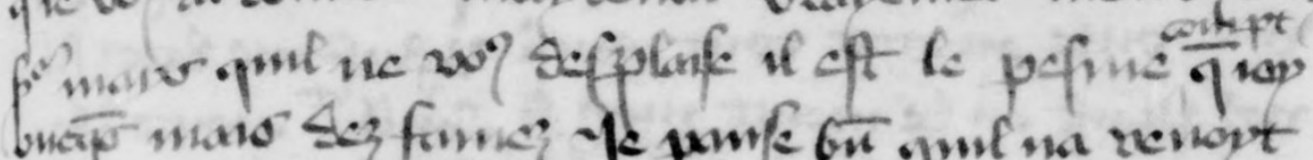

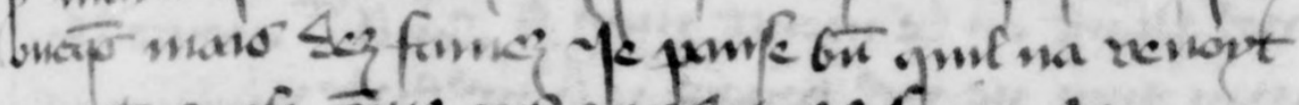

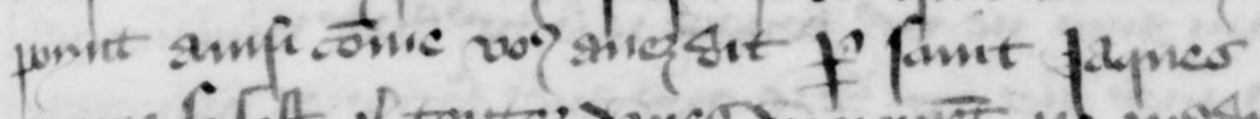

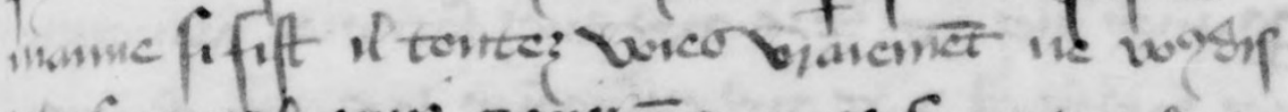

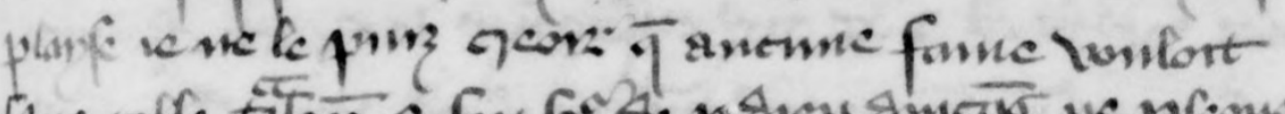

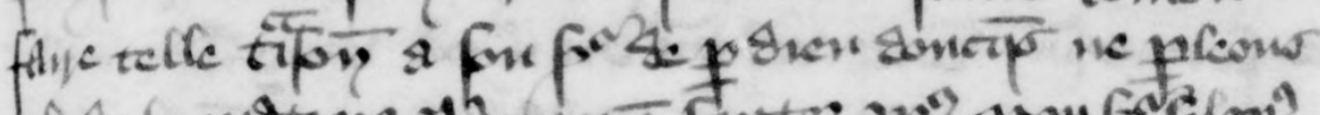

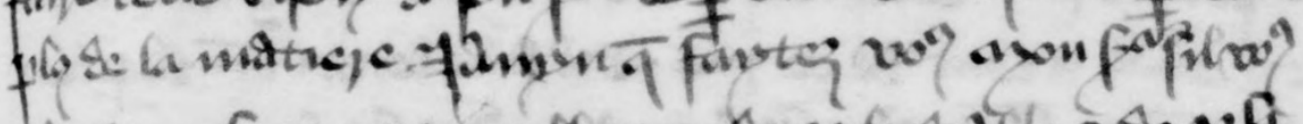

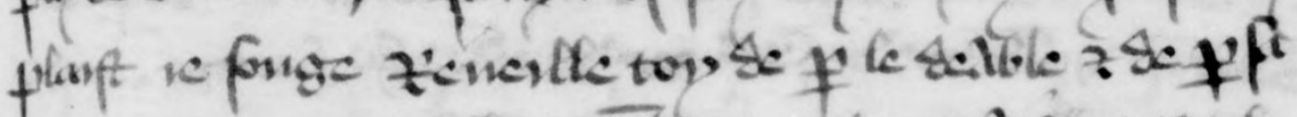

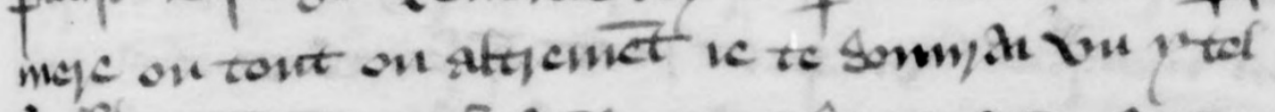

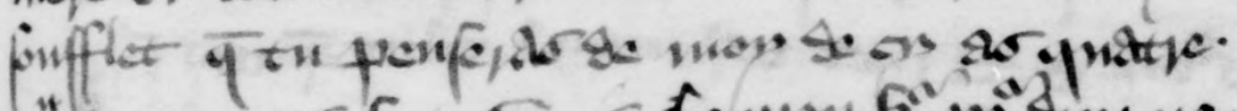
is

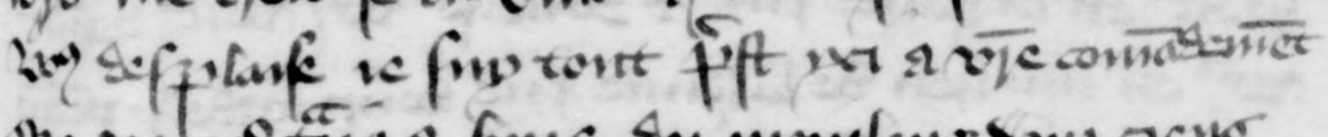

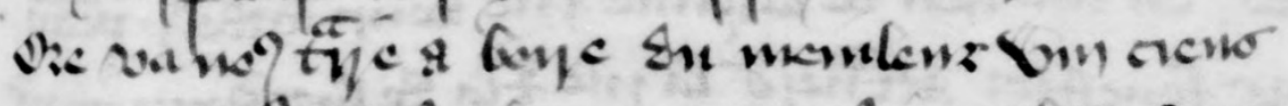

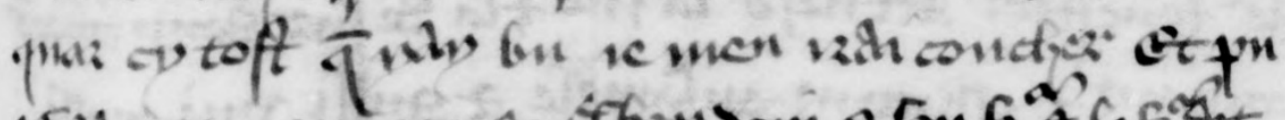

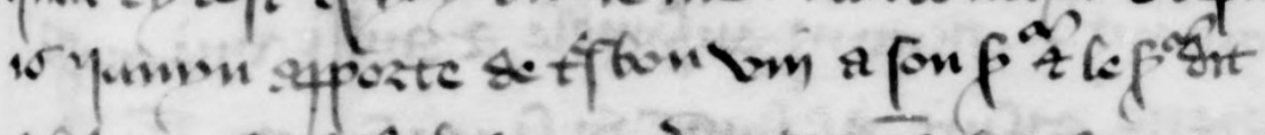

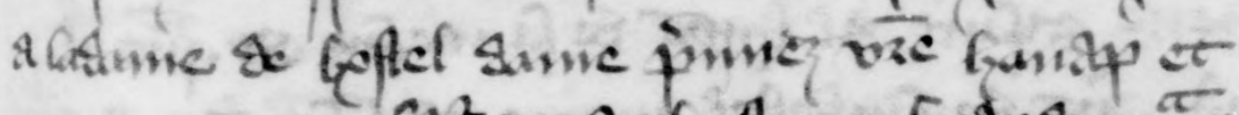

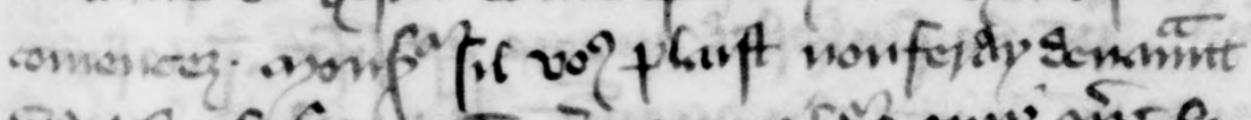

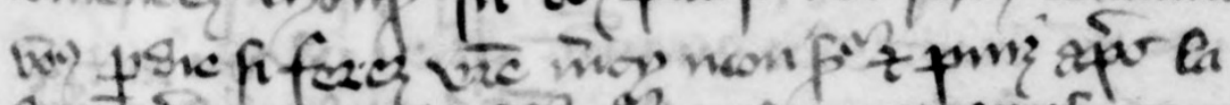

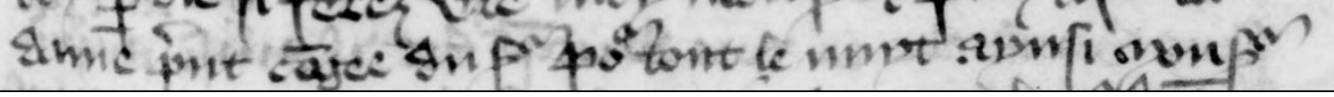

Figure 1 : Manières de langage 1396, in Opuscula grammatica, Ms. Paris, BnF, nouv. acq. lat. 699, f. 123r (détail). (C) Source Gallica.bnf.fr, Bibliothèque nationale de France. 
Comme on le voit, la répartition des tours par l'éditeur donne deux tours consécutifs au vénitien et résulte en une erreur sémantique : dans la logique de l'édition (i.e. du retour à la ligne et du tiret cadratin, symbolisant le changement de locuteur), le français aurait alors reposé la question de l'origine et le vénitien devrait en ce cas répondre d'une manière absurde (« je vien tout droit de Lumbardie » $\mathbf{V}$ «Verament, sir, je su de France »).

Avant de nous pencher sur cette mauvaise attribution, il convient tout d'abord de rappeler le statut physique de ce texte : ce qui nous apparaît comme une simple succession de tours bien distingués les uns des autres doit en réalité être ramené à la forme du document. Dans le seul témoin qu'il nous ait été permis de consulter, les tours de parole s'enchaînent de façon linéaire et la plupart du temps sans marques graphiques ${ }^{16}$. On comprend alors qu'une des fonctions des marqueurs d'oralité situés à l'initiale des tours ne réside pas dans une quelconque représentation de l'oral mais sert des impératifs pratiques : en démarquant les passages, ils permettent une lecture plus aisée, surtout si l'on se rappelle qu'il s'agit de documents didactiques, destinés par conséquent à une lecture performée.

Il n'est pas dans notre intention de critiquer ici l'édition de ce texte mais plutôt d'essayer de comprendre la cause de cette erreur d'attribution d'un tour. Nous faisons l'hypothèse que cette méprise est liée au «Et » de relance interphrastique situé en première position de la phrase : son positionnement a de fait amené l'éditeur à en faire un tour isolé, au mépris de la logique. En

d'autres termes, une erreur mineure nous ré-

vèle un fait crucial : la preuve de l'efficacité certaine des marqueurs d'oralité comme outils indiquant l'organisation des tours de parole. Il ne s'agit ici, en réalité, que d'une sur-détermination du rôle d'un élément.

\section{Conclusion}

La manière de faire dont nous avons souhaité illustrer le fonctionnement et l'assise diffère principalement des autres modèles par ce recours à une intuition ou un investissement du chercheur que l'on ne masquerait pas par des chiffres, ainsi que par une réflexion sur ce qui, au départ, nous entraîne irrémédiablement à considérer différentes parties d'un texte comme " orales ». De façon anticlimatique, elle s'intéresse toutefois à peu près aux mêmes catégories (interjections, lexèmes pragmatiques, phénomènes syntaxiques, etc.) et reprend bien souvent des conclusions déjà énoncées.

L'intérêt nous semble plutôt résider dans ce léger déplacement de la question et la capacité à fonctionner de concert avec ces autres méthodes. En croisant les

\footnotetext{
${ }^{16}$ Le folio du manuscrit reproduit à titre d'exemple d'absence de marques graphiques dans la figure 1 ne correspond pas au passage cité de l'édition de Kristol (1995), en raison de l'absence de certains passages dans ce manuscrit français.
} 
résultats, nous ne pouvons qu'arriver à une appréhension plus juste de ce qui, inévitablement, nous demeurera toujours perdu.

L'épistémologie qui soutient notre approche se partage donc entre l'étude ergonomique des systèmes de signes (affordances, contraintes d'optimalités), les imaginaires induits par ces propriétés mais aussi une attention historique pour des sources que l'on aurait tort de considérer comme de simples réservoirs de données. Notre but ne concerne en aucun cas une reconstruction de la langue parlée mais se rapproche peut-être davantage de l'état de lucide incertitude préconisée par Zumthor (1987: 18) :

Recherches et réflexions sur l'oralité de la chanson de geste [...] ont eu jusqu'ici pour effet d'ébranler quelque peu les assurances, d'atténuer la portée de plusieurs termes et de diffuser un petit nombre de doutes communs. Elles ne nous ont point apporté de certitude. Mais, justement, la question n'est pas celle d'une certitude. C'est celle de notre mode de perception et, plus encore, de notre volonté d'ouverture, impliquant une intégration, dans la lecture de nos vieux textes, d'une sorte d'imagination critique.

Il nous semble fondamental de ne pas considérer les marqueurs d'oralité comme devant à tout prix " représenter " un oral. La construction d'une catégorie ad hoc est déjà le signe de l'engagement du chercheur, et l'empêche de céder au démon de la reconstruction. Il y a, dans l'appréhension de cette catégorie et de l'effet d'oral, quelque chose de saint Jean découvrant, à la fin de son évangile, le tombeau du Christ. Il nous est en effet alors dit : "Il vit et il crut » (Jean, $20: 8$ ). Mais il faudrait toujours se rappeler que ce que l'apôtre observe à ce moment-là, c'est un tombeau vide.

\section{RÉFÉRENCES BIBLIOGRAPHIQUES}

\section{a) Corpus et textes cités}

BÉNICHOU-SAMSON, Édouard [éd.] (2016) : Le rôle de l'Homme pécheur (BnF, ms., naf 6514), thèse pour le diplôme d'archiviste-paléographe, Ecole nationale des chartes.

BlanCHARD, Joël [éd.] (2012) : Procès de Jacques d'Armagnac. Genève, Droz.

Du FAIL, Noël (1994 [1547]) : Propos rustiques, éd. Gabriel-André Pérouse et Roger Dubuis. Genève, Droz.

$\mathrm{BFM}=$ Base de français médiéval, ENS de Lyon, http://txm.bfm-corpus.org

LA Vigne, Andrieu de (1979 [1496]) : Le Mystere de Saint Martin (1496), éd. André Duplat. Genève, Droz.

KRISTOL, Andres M. [éd.] (1995). Manières de langage (1396, 1399, 1415), Londres : Anglo-Norman Text Society. 
Opuscula grammatica, Ms. Paris, BnF, nouv. acq. lat. 699, f. 123r, Gallica.bnf.fr, Bibliothèque nationale de France, URL: https:/gallica.bnf.fr/ark:/12148/btv1b10035413k/f6.item.r=699\#

PÉGEOT, Pierre, Odile DERNIAME \& Madeleine HÉNIN [éds] (2013) : Les lettres de rémission du duc de Lorraine René II (1473-1508). Turnhout, Brepols.

RabELAIS, François (1955) : Euvres complètes, éd. Jacques Boulenger. Paris, Gallimard.

RYCHNER, Jean [éd.] (1999) : Les.XV. joies de mariage. Genève, Droz.

SMiTH, Darwin [éd.] (2002) : Maistre Pierre Pathelin. Le miroir d'orgueil: texte d'un recueil inédit du XVe siècle (mss Paris, B.N.F. fr. 1707 et 15080). Saint-Benoît-du-Sault, Tarabuste.

TisseT, Pierre [éd.] (1960): Procès de condamnation de Jeanne d'Arc, vol. 1-3. Paris, Klincksieck.

\section{b) Études}

ADAM, Jean-Michel (2011) : La linguistique textuelle. Introduction à l'analyse textuelle des discours. Paris, Armand Colin.

ANHEIM, Étienne (2020) : "Rapport introductif ", in La voix au Moyen Âge. Actes du $50^{\circ}$ congrès de la SHMESP. Paris, Éditions de la Sorbonne, 2020, 11-34.

BARTHES, Roland (1984) : Le bruissement de la langue. Paris, Seuil.

BERRENDONNER, Alain (2004) : "Grammaire de l'écrit vs grammaire de l'oral : le jeu des composantes micro- et macro-syntaxiques ", in Alain Rabatel (éd.), Interactions orales en contexte didactique. Lyon, Presses universitaires de Lyon, 249-264.

BLANCHE-BENVENISTE, Claire \& Colette JEANJEAN (1987) : Le français parlé: transcription et édition. Paris, Didier érudition.

CISLARU, Georgeta \& Thierry OLIVE (2018) : Le processus de textualisation: analyse des unités linguistiques de performance écrite. Louvain, De Boeck Supérieur.

CULPEPER, Jonathan \& Merja KYTÖ (2010) : Early Modern English Dialogues. Spoken Interaction as Writing. Cambridge, Cambridge University Press.

DERRIDA, Jacques (1967) : De la grammatologie. Paris, Minuit.

FLECK, Ludwik (2008 [1935]) : Genèse et développement d'un fait scientifique. Paris, Flammarion.

GAUVARD, Claude (1991) : " De grace especial »: crime, état et société en France à la fin du Moyen Age. 2 vols. Paris, Publications de la Sorbonne.

GIBSON, James J. (1977) : "The Theory of Affordances », in Robert Shaw \& John Bransford (éds), Perceiving, Acting and Knowing. Toward an Ecological Psychology. Hillsdale, Lawrence Erlbaum Associates, 67-83. 
GLIKMAN, Julie \& Nicolas MAZZIOTTA (2013) : «Représentation de l'oral et structures syntaxiques dans la prose de la Queste del saint Graal (1225-1230) ", in Dominique Lagorgette \& Pierre Larrivée (éds.), Représentations du sens linguistique V, Chambéry, Presses universitaires Savoie Mont Blanc, 69-90, URL: https://halshs.archives-ouvertes.fr/halshs-01073928

Guerreau-JALABERT, Anita (1997) : «Parole/Parabole. La parole dans les langues romanes: analyse d'un champ lexical et sémantique", in Rosa Maria Dessi \& Michel Lauwers (éds.), La parole du prédicateur (Ve-XVesiècle). Turnhout, Brepols, 311-339.

Guillot, Céline (2009) : "Ecrit médiéval et traces d'oralité : l'exemple de l'adverbe or(e) », in Eva Havu (éd.), La langue en contexte. Helsinki, Société Néophilologique, 267-281.

JACOBS, Andreas \& Andreas H. JUCKER (1995) : " The historical perspective in pragmatics ", in Andreas H. Jucker (éd.). Historical Pragmatics: Pragmatics Developments in the History of English. Amsterdam, John Benjamins, 3-36.

JUCKER, Andreas H. (2015) : «Pragmatics of fiction : Literary uses of uh and um». Journal of Pragmatics, 86, 63-67.

KoCH, Peter \& Wulf OeSTERREICHER (2001) : "Langage parlé et langage écrit », in Lexikon der Romanistischen Linguistik, I/2. Tübingen, Niemeyer, 584-627.

KRISTOL, Andres M. (1993) : "Que dea! Mettes le chapron, paillard, com tu parles a prodome". La représentation de l'oralité dans les Manières de langage du XIV $-\mathrm{XV}^{\mathrm{e}}$ siècle ». Romanistiches Jahrbuch, 43, 35-64.

LEFEUVRE, Florence \& Noalig TANGUY (2012) : «La représentation de l'oral dans les dialogues dramaturgiques : les énoncés averbaux ", in Claire Despierres \& Mustapha Krazem (éd.), Quand les genres de discours provoquent la grammaire... et réciproquement. Limoges, Lambert-Lucas.

LETT, Didier (2008) : Un procès de canonisation au Moyen Âge : essai d'histoire sociale. Nicolas de Tolentino, 1325. Paris, Presses Universitaires de France.

LORAUX, Nicole (1993) : "Éloge de l'anachronisme en histoire ». Le genre humain, 27, 23-39.

MAHRER, Rudolf (2014) : Lire l'oral. Pour une typologie linguistique des représentations écrites de l'oralité (le cas du français). Thèse de doctorat sous la direction de Jacqueline Authier-Revuz et de Gilles Philippe. Paris 3 - Sorbonne nouvelle.

MARCHELLO-NIZIA, Christiane (2014) : "L'importance spécifique de l'oral représenté pour la linguistique diachronique ", in Wendy Ayres-Bennett \& Thomas M. Rainsford, L'histoire du français. État des lieux et perspectives. Paris, Classiques Garnier, 161-174.

MARCHELlO-NiziA, Christiane (2017) : «Les débuts de l'oral représenté en français: marquage du discours direct dans les plus anciens textes ", in Claire Badiou-Monferran, Samir Bajric \& Philippe Monneret, Penser la langue. Sens, texte, histoire. Hommages à Olivier Soutet. Paris, Honoré Champion, 117-129.

MORSEL, Joseph (2000) : «Ce qu'écrire veut dire au Moyen Âge... Observations préliminaires à une étude de la scripturalité médiévale ». Memini, 1, 3-43.

OfFENSTADT, Nicolas (2013) En place publique. Jean de Gascogne, crieur au XVe siècle. Paris. 
Stock.

OPPERMANN-MARSAUX, Evelyne (2011) : " Les emplois du marqueur discursif di va en ancien français ». Discours, 8. DOI : https://doi.org/10.4000/discours.8321

PARUSSA, Gabriella (2018) : «La représentation de l'oral à l'écrit et la diachronie du français. Un nouveau projet de recherche ", in Wendy Ayres-Bennett et al., Nouvelles voies d'accès au changement linguistique. Paris, Classiques Garnier, 181-199.

RODRÍGUEZ-SOMOLINOS, Amalia (1995) : "Certes, voire: l'évolution sémantique de deux marqueurs assertifs de l'ancien français ». Linx $, 32,51-76$.

RODRÍGUEZ-SOMOLINOS, Amalia (2016) : «Présentation ». Linx, 73 [Dossier Énonciation et marques d'oralité dans l'évolution du français], 9-16, DOI : https://doi.org/10.4000/linx. 1620

ROUSSEAU, Jean-Jacques (1990 [1781]) : Essai sur l'origine des langues, éd. Jean Starobinski. Paris, Gallimard,

SCHEGLOFF, Emanuel A. (1982) : «Discourse as an Interactional Achievement: Some Uses of uh huh and other things that come between sentences ", in Deborah Tannen, Analyzing Discourse: Text and Talk. Washington, Georgetown University Press, 71-94.

SCHMID, Anne-Françoise \& Muriel MAMBRINI-DOUDET (2019) : Épistémologie générique. Paris, Kimé.

SCHOURUP, Lawrence (1999) : «Discourse markers ». Lingua, $107:$ 3, 227-265.

SHORT, Mick et al. (1997) : "A (free direct) reply to Paul Simpson's discourse ». Journal of Literary Semantics, $26: 3,219-228$.

SMITH, Darwin ; Gabriella PARUSSA \& Olivier HALÉVY (2014) : Le théâtre français du Moyen Âge et de la Renaissance: histoire, textes choisis, mises en scène. Paris, L'Avant-scène théâtre.

TAVIANI, Ferdinando (1969) : La commedia dell'arte e la società barocca, Roma, Bulzoni.

VERMANDER, Pierre (à paraître) : « Pour une pragmaphilologie. Marqueurs d'oralité et variation dans les différents témoins de Maistre Pierre Pathelin ". Romania.

VINCENSINI, Jean-Jacques (2006) : « Formes et fonctions structurantes. À propos de quelques interjections en ancien et en moyen français ». Langages, 161, 101-111.

WAHNICH, Sophie (2009) : Les émotions, la Révolution française et le présent. Exercices pratiques de conscience historique. Paris, CNRS.

WLODARCZYK, Matylda (2005) : «Is Reanimation of Voices Possible? Pragmatics of Reported Speech in Selected Middle English Texts ». Studia Anglica Posnaniensa, 41, 99-113.

ZUMTHOR, Paul (1987) : La lettre et la voix: de la "littérature " médiévale. Paris, Seuil. 